ARTICLE

Received 27 May 2015 | Accepted 21 Sep 2015 | Published 26 Nov $2015 \quad$ DOl: 10.1038/ncomms9681 OPEN

\title{
An in cellulo-derived structure of PAK4 in complex with its inhibitor Inka1
}

Yohendran Baskaran ${ }^{1, \star}$, Khay C. Ang ${ }^{1,2, \star}$, Praju V. Anekal ${ }^{1}$, Wee L. Chan ${ }^{1}$, Jonathan M. Grimes ${ }^{3,4}$, Ed Manser ${ }^{1,5,6}$ \& Robert C. Robinson ${ }^{1,2}$

PAK4 is a metazoan-specific kinase acting downstream of Cdc42. Here we describe the structure of human PAK4 in complex with Inka1, a potent endogenous kinase inhibitor. Using single mammalian cells containing crystals $50 \mu \mathrm{m}$ in length, we have determined the in cellulo crystal structure at $2.95 \AA$ resolution, which reveals the details of how the PAK4 catalytic domain binds cellular ATP and the Inka1 inhibitor. The crystal lattice consists only of PAK4PAK4 contacts, which form a hexagonal array with channels of $80 \AA$ in diameter that run the length of the crystal. The crystal accommodates a variety of other proteins when fused to the kinase inhibitor. Inka1-GFP was used to monitor the process crystal formation in living cells. Similar derivatives of Inka1 will allow us to study the effects of PAK4 inhibition in cells and model organisms, to allow better validation of therapeutic agents targeting PAK4.

\footnotetext{
${ }^{1}$ Institute of Molecular and Cell Biology, A*STAR (Agency for Science, Technology and Research), Biopolis, Proteos Building, 61 Biopolis Drive, 8-15, Singapore 138673, Singapore. ${ }^{2}$ Department of Biochemistry, Yong Loo Lin School of Medicine, National University of Singapore, Singapore 117597, Singapore. ${ }^{3}$ Division of Structural Biology, Wellcome Trust Centre for Human Genetics, University of Oxford, Roosevelt Drive, Oxford OX3 7BN, UK. ${ }^{4}$ Diamond Light Source Ltd., Diamond House, Harwell Science and Innovation Campus, Didcot, Oxfordshire OX11 ODE, UK. ${ }^{5}$ Institute of Medical Biology,

A*STAR, Biopolis, Singapore 138648, Singapore. ${ }^{6}$ Department of Pharmacology, Yong Loo Lin School of Medicine, National University of Singapore, Singapore 117597, Singapore. ${ }^{\star}$ These authors contributed equally to this work. Correspondence and requests for materials should be addressed to E.M. (email: ed.manser@imcb.a-star.edu.sg).
} 
$\mathrm{M}$ ammalian PAK isoforms are categorized into two groups on the basis of their structural and biochemical features: the conventional or group I PAKs in human comprise PAKs1-3, whereas the group II PAKs (PAK4-6) are encoded by three genes in mammals ${ }^{1-3}$. PAK4-like kinases are ubiquitously expressed in metazoans but are not found in protozoa or fungi ${ }^{4,5}$. This is consistent with PAK4 functioning primarily at cell-cell contacts in mammalian cells ${ }^{6}$, with Cdc42 also being required for adherent junction formation ${ }^{7}$. The phenotype of PAK4-null mice, which is embryonic lethal, involves defects in the fetal heart as well as in neuronal development and axonal outgrowth ${ }^{8}$. The loss of PAK4 prevents proper polarization and thus formation of the endothelial lumen ${ }^{9}$, consistent with defects seen in PAK4 - / mice ${ }^{10}$.

PAK4 is a kinase with strong links to cellular transformation and cancer metastasis, as reviewed ${ }^{11}$. The structural basis for PAK4's preference for serine containing substrate sites has recently been elucidated ${ }^{12}$. We have shown that Cdc42 directly regulates PAK4 activity in mammalian cells through an autoinhibitory domain (AID) that binds in a manner similar to pseudosubstrates ${ }^{13,14}$. This is consistent with the notion that PAK4 lacking residues $10-30$ in the $\mathrm{Cdc} 42 / \mathrm{Rac}$ interactive binding domain is active ${ }^{15}$. Although PAK1 activation in vivo occurs through activation loop Thr- 423 phosphorylation ${ }^{16}$, it is notable that PAK4 is constitutively phosphorylated on Ser-474 (ref. 13) and kept in check through the intramolecular association of the AID. The binding of Cdc42 can serve to activate PAK4 in cells, but it is unclear whether there is any autophosphorylation event associated with this activation ${ }^{13}$. As PAK4 does not appear to use adaptors, we investigated the possibility that Inka1, first identified as a PAK4-binding protein in frogs ${ }^{17}$, might fulfill this role.

In-vivo protein crystallization is rare with mammalian examples including insulin ${ }^{18}$ and Charcot-Leyden crystals ${ }^{19,20}$. The observation that haemoglobin could crystallize on dilution of unpurified red cell lysate ${ }^{21}$ facilitated the advent of protein X-ray crystallography ${ }^{22}$. Only recently have microcrystals generated inside bacterial or insect cells become amenable to X-ray analysis ${ }^{23-25}$. A coral fluorescent protein that forms diffractionquality micron-sized crystals within mammalian cells ${ }^{26}$ indicates the mammalian cell environment could be a suitable host for a number of proteins, which are not normally crystalline.

Experiments described here suggest that Inka proteins are in fact endogenous inhibitors of PAK4, with the two human Inka isoforms sharing a high degree of sequence identity in the region previously termed the Inca box ${ }^{17}$. Inkal contains an additional PAK4 inhibitory sequence at its carboxy terminus and either of these sequences can promote crystallization of the catalytic domain of human PAK4 in mammalian cells. An in-cellulo protein structure, from X-ray experiments on single crystals formed within a mammalian cell, reveals a hexagonal array of PAK4 subunits that was suggestive of an ability to accommodate other proteins in the lattice. This was demonstrated by fusing Inkal to green fluorescent protein (GFP). Because of these features the PAK4 array has potential as a protein analogue of 'crystalline molecular flasks' in which guest molecules can reside to facilitate their X-ray analysis ${ }^{27}$.

\section{Results}

Inka1 is an endogenous PAK4 inhibitor. We previously reported that the Cdc42 effector PAK4 is regulated by an AID (Fig. 1a), which serves to control the constitutively phosphorylated catalytic (PAK4cat) domain ${ }^{13}$. Although Cdc42 upregulates PAK4 activity in vivo, this kinase activation cannot be observed using recombinant proteins in vitro ${ }^{14}$, indicating other protein(s) might be involved. Indeed, it has been suggested that Src SH3 domain interaction with the core AID sequence might be an alternate means of regulating $\mathrm{PAK} 4^{14}$, although a cellular Src-PAK4 interaction has not been detected. There are few PAK4-interacting proteins known other than the Cdc42-like GTPases ${ }^{4}$. One Xenopus PAK4-binding protein originally identified through a yeast two-hybrid screen is a $30-\mathrm{kDa}$ neural crest enriched protein termed Inka1 (previously Inca ${ }^{28,29}$ ), although the role of this putative adaptor was not determined. The protein is also designated FAM212a and FAM212b in the protein database based on their common central 38 amino acid sequence (166-203) here termed the Inka box (iBox, Fig. 1a).

We decided to investigate the role of human Inka1 by further testing its ability to bind to various PAK4 constructs in mammalian cells. Inkal bound to an activated PAK4 with a mutated AID (designated PAK4*) significantly better than wildtype PAK4 (Fig. 1b). This suggested that the PAK4 AID limits Inkal access to the PAK4 catalytic domain (Fig. 1b) with which it interacts ${ }^{29}$. The recombinant 38 amino acid 'Inka box' (GSTiBox) is a potent of PAK4cat inhibitor in vitro (Fig. 1c) but does not affect PAK1, suggesting Inka1 is a specific group II $\mathrm{PAK}^{30}$ inhibitor. Inka1 probably acts also on PAK5 and PAK6, as their substrate binding pockets are essentially identical ${ }^{31}$. In-vitro measurements indicate GST-Inka1 has a Ki of $30 \mathrm{nM}$ (Fig. 1d), which is comparable with the avidity of protein kinase A (PKA) inhibitor $(\mathrm{PKI})^{32}$. The iBox sequence (Fig. 1a) contains the tripeptide PLV in common with the PAK4-AID, which binds in the substrate-docking site ${ }^{14,33}$.

Inka1 has two functional inhibitory regions. Intriguingly, we noted that the inhibitory iBox appears to be duplicated in the C-terminal 22 amino acids of Inkal (Figs 1a and 2c), which we term iBox-C. Synthetic 24 mer peptides, corresponding to the amino- or C-terminal 2/3rd of the iBox or the iBox-C, exhibited $\mathrm{Ki}$ values of $0.2-0.4 \mu \mathrm{M}$ (Fig. $1 \mathrm{~d}$ ), which suggested that all 38 amino acids centred on the PLV motif are involved in PAK4 inhibition. Thus, Inkal functions as an inhibitor of kinase activity; given that it lacks sequence conservation outside these PAK4 inhibitory motifs (the iBox or iBox-C) it seems likely to be that the main function of the protein is to negatively regulate PAK4 activity. Deletion of either Inka1 or Inka2 cause subtle defects in frog and mouse development ${ }^{28,29}$, not inconsistent with human Inkal being causative in a chromosomal microdeletion being associated with cleft lip and the central nervous system abnormalities $^{34}$. Inkal is expressed in a number of cell types in the early mouse embryo ${ }^{28}$.

Inkal forms crystals with PAK4 in cells. We asked whether Inka1 and PAK4 co-localize in mammalian cells (Fig. 2a). Inka1 alone is predominantly nuclear but PAK4 is not. However, co-expressing PAK4, which has been reported to contain an $\mathrm{N}$-terminal nuclear localization signal ${ }^{35}$, redistributed Inka1 into the cytoplasm. This is interesting given the established role of PKI in terminating nuclear but not cytoplasmic PKA signals ${ }^{36}$. We next tested whether Inkal inhibits active PAK4cat in vivo. Unexpectedly, the co-expression of these proteins consistently yielded cytoplasmic protein crystals that contained both Inka1 and PAK4, judged by immunostaining (Fig. 2b). By phasecontrast microscopy, these often appear as single elongated crystals $>50 \mu \mathrm{m}$ that extend across the cytoplasm (Fig. 2b, boxed region). Curiously, many truncated Inka1 constructs were capable of forming crystals with PAK4cat, when these contained either the central iBox or iBox-C (Fig. 2c). These crystals look remarkably similar (Supplementary Fig. 1), suggesting they have 


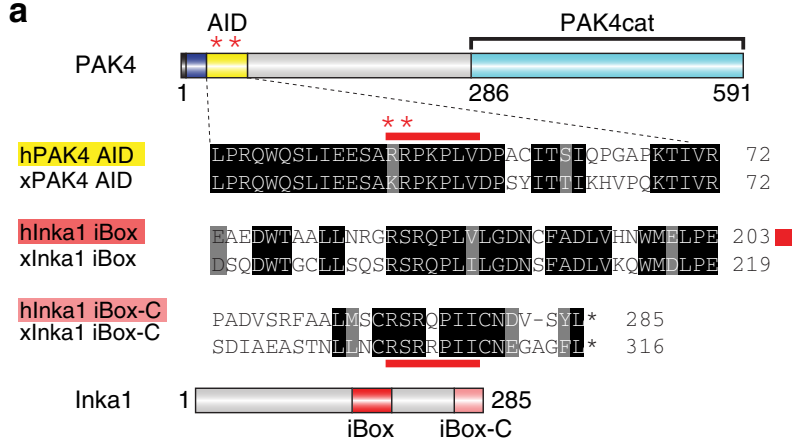

b

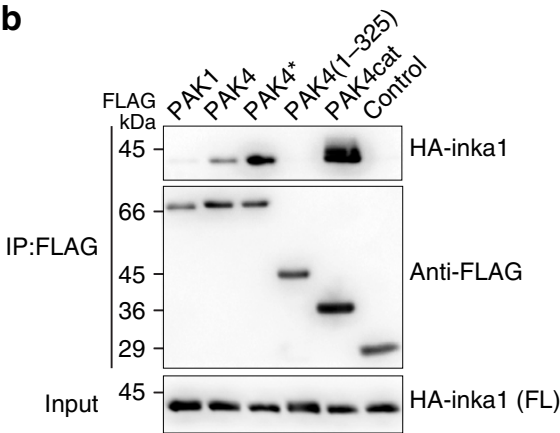

c
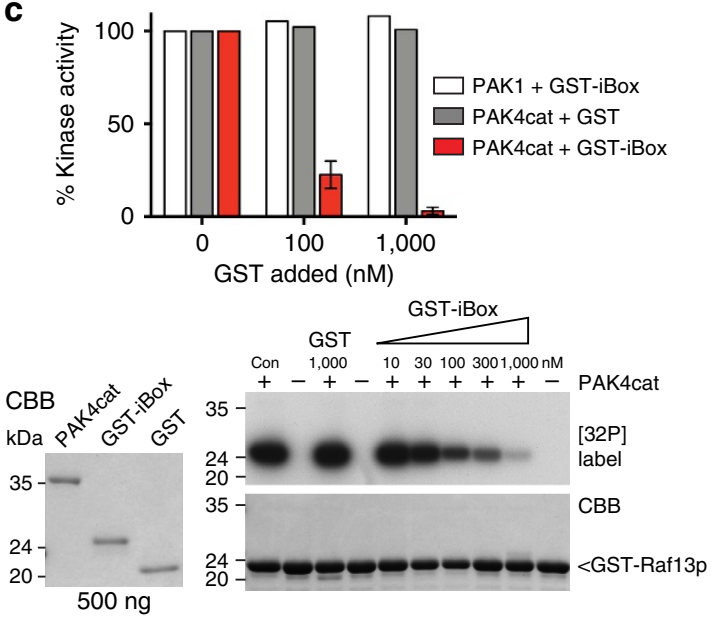

d

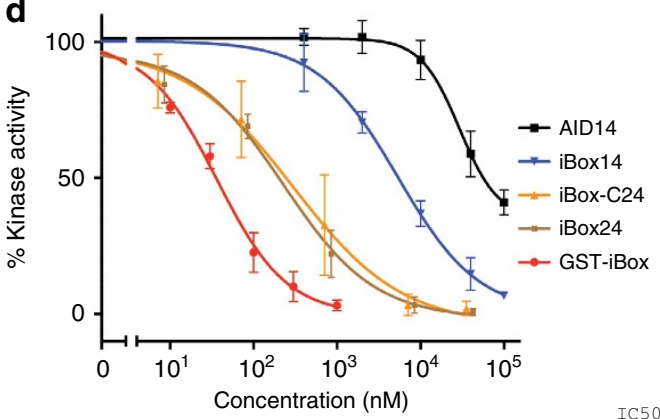

iBox IC50 BBox24 AEDWTAALINRGRSROPLVLGDNC AEDWTAALLNRGRSRQPLVLGDNC $229 \mathrm{nM}$ $\begin{array}{cc}\text { RSRQPLVLGDNCFADLVHNWMELP } & 382 \mathrm{nM} \\ \text { iBox-C24 VSRFAALMSCRSROPIICNDVSYL * } & 300 \mathrm{nM}\end{array}$ BBox14 PAK4 AID14

Figure 1 | Inka1 is a potent kinase inhibitor. (a) PAK4 architecture and alignment of the AID and the Inka1 iBox and iBox-C from frogs and human. Red asterisks indicate activation mutations in PAK4* (RR48/49AE). Red bars indicate pseudosubstrate sequences. (b) Co-immunoprecipitation of full-length HAInka1 by FLAG-tagged PAK4 constructs. (c) Kinase assays using 6His-PAK1 (activated) or PAK4cat, with GST-iBox as indicated. Activity was assessed by the phosphorylation of GST-Raf13 quantified by densitometry (lower right). The quality of the purified proteins is indicated (lower left). (d) The inhibition profile of GST-iBox and selected peptides of the iBox and iBox-C $\left(n=3\right.$, error bars indicate s.e.m.). The $I_{50}$ values were determined from the intercepts of the graphs.

the same underlying organization. Inkal constructs that contain both copies of the PAK4 inhibitory regions (residues 165-285) were most efficient at inducing crystals. The C-terminal 31 amino acid of Inkal (255-285) was able to induce crystals more efficiently than the Inkal (166-203) when they are expressed as haemagglutinin (HA)-tagged proteins, although the iBox38 has a higher affinity in vitro. To confirm that these crystals indeed contain a 1:1 ratio of both components, we generated a singlechain Flag-iBox-PAK4cat construct as illustrated in Fig. 2c. This expression construct yielded abundant in-cellulo crystals in multiple human cell types.

The in-cellulo structure of Inka1 bound to PAK4cat. As the crystals of PAK4 appeared to be relatively stable within the cell, we decided not to attempt to purify these further. To tackle the incellulo crystal structure of iBox-PAK4cat, intact monkey COS-7 cells that contained large single needle crystals $(<5 \mu \mathrm{m}$ in crosssection by $50-100 \mu \mathrm{m}$ ) were trypsinized to yield rounded cells in which large crystals could be easily observed (Fig. $2 \mathrm{~d}$, arrows). The cells containing the largest crystals were individually mounted in cryoloops and flash frozen (Fig. 2e). These crystals were exposed to $\mathrm{X}$-rays on the Diamond synchrotron microfocus beamline I24 equipped with microapertures. Typical diffraction data are given in Supplementary Fig. 2, which illustrate the importance of this micro beam to the quality of data. The merged data from five crystals led to the structure being solved at $2.95 \AA$ resolution (Fig. 3a), the statistics for which are given in Table 1 . To our knowledge, this is the first in-cellulo crystal structure of a mammalian protein to be elucidated within intact mammalian cells.

The X-ray structure of these in-cellulo crystals provided us with a number of important insights: under cellular conditions, PAK4cat adopts a typical 'closed' active kinase conformation that includes ATP bound to two magnesium ions. As we expected, the activation (A) loop Ser474 is phosphorylated and the central region of the iBox is packed against the kinase through both main-chain and side-chain interactions (Fig. 3a). The side chain of PAK4 Arg359, which lies at the end of the $\alpha \mathrm{C}$ helix, stabilizes the catalytic competent state by interacting with the phospho-Ser474. When the $\mathrm{N}$-lobe $\alpha \mathrm{C}$ helix is held in such a 'closed' state with respect to the C-lobe, it allows for proper coordination of bound ATP. $2 \mathrm{Mg}^{2}+$ for catalytic transfer. Most structures with or without substrates bound ${ }^{12}$ show a coupling between Arg359 and the Ser474 phosphate: the phosphorylated PAK1 Thr423 appears to use the same A-loop to phosphate coupling, to stabilize the $\alpha C$ helix in an active state ${ }^{16}$. Indeed, such coupling may well be a common mechanism feature of kinases in which activation loop phosphorylation is essential for activity, for example, $\mathrm{PKA}^{37}$.

On the basis of these experiments, we hypothesize that Inka1 stabilizes the ATP-bound crystallization-competent conformation of the kinase domain by preventing ATP hydrolysis through binding tightly in the cleft between the $\mathrm{N}$ - and C-lobes. This in-cellulo iBox-PAK4cat structure determined in space group $\mathrm{P}_{3}$ was verified by comparison with the structure of the complex determined at $2.0 \AA$ resolution from $\mathrm{P}_{1} 2_{1} 2$ crystals grown 


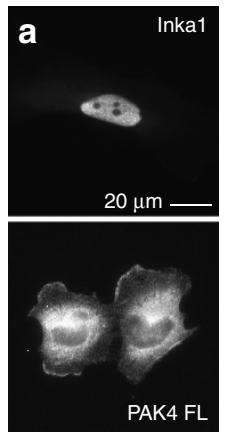

b
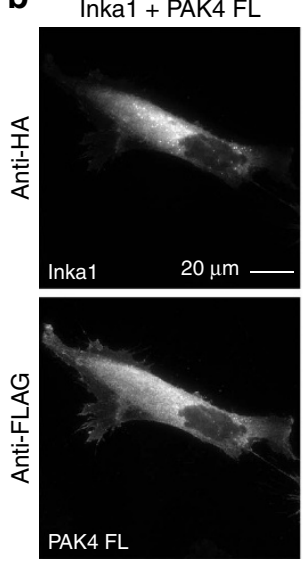

c

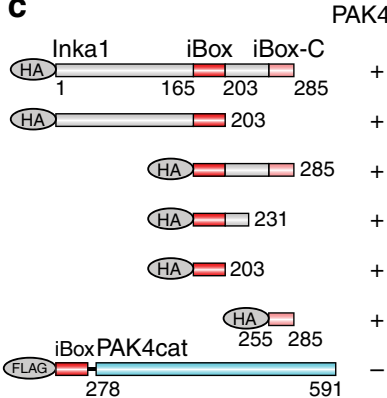

Inka1 + PAK4cat
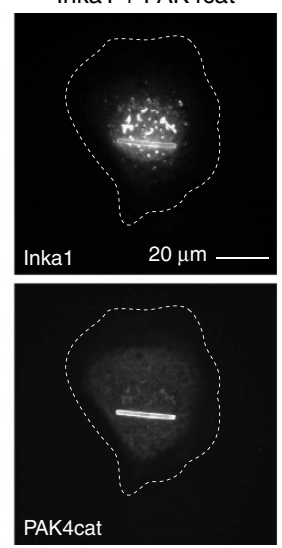

Inka1 + PAK4cat

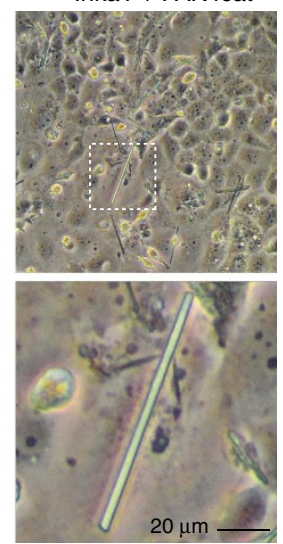

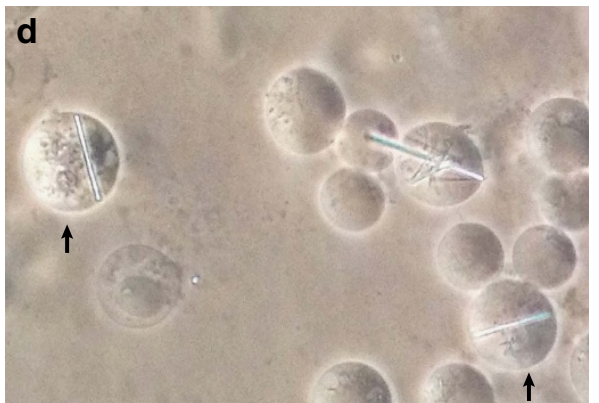

In cellulo crystals, trypsinized cells

e

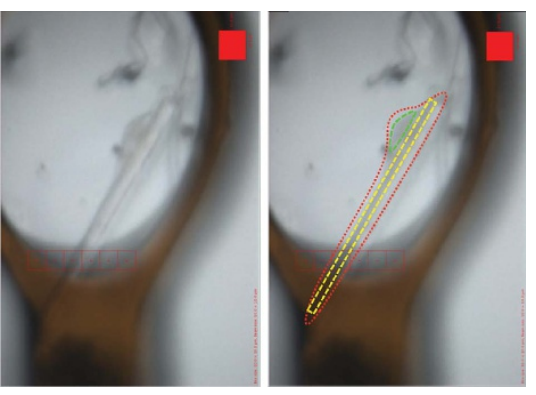

In cellulo crystal, on cryoloop

Figure 2 | Intracellular PAK4cat:Inka1 crystals. (a) Inka1 and PAK4 show nuclear and cytoplasmic localization, respectively. (b) Co-expression leads to cytoplasmic enrichment of Inka1 (left panels). Inka1 and PAK4cat co-expression results in intracellular crystals (right panels), which immunostain for both proteins (middle panels). (c) Inka1 regions capable of generating co-crystals. A single chain fusion of iBox-PAK4cat efficiently generated intracellular crystals. (d) In-cellulo crystals of trypsinized cells. (e) A single cell mounted on a cryo-loop on a synchrotron beamline. The crystal (yellow), the cell membrane (red) and the nucleus (green) are highlighted.

in vitro from purified PAK4cat and a synthetic iBox $24 \mathrm{mer}$ peptide (Fig. 3b). These two structures are essentially identical, although more of the Inkal backbone is visible in the in-cellulo structure and in vitro structure lacks bound ATP and $\mathrm{Mg}^{2+}$. We are able to determine the side-chain disposition of 28 of the 38 iBox amino acids; the relative close disposition of the visible $\mathrm{N}$ and $\mathrm{C}$ termini suggest the remaining residues make intramolecular contacts to stabilize the Inkal inhibitor in a loop-like manner. This hypothesis is consistent with the relative Ki of the various Inkal peptides shown in Fig. 1.

The main chain and side chains of Inkal residues 171-196 are clearly visible with the C-terminal F191-N197 forming a helix that packs against the C-lobe (Fig. 3b). This interaction primarily involves the packing of hydrophobic side chains of Inka1, including F191, L194 and V195 against the end of the C-lobe helix $\alpha$-EF and Arg488. It is likely to be that these interactions provide kinase specificity, as this region is in general more diverse. Interestingly, this part of the PAK1 C-lobe including both helix $\alpha$ $\mathrm{EF}$ and $\alpha-\mathrm{G}$ makes extensive contacts with its $\mathrm{AID}^{38}$, which can inhibit Pak1 with $20 \mathrm{nM}$ affinity (in trans). Unlike Inka1, the PAK1 AID makes no contacts with the substrate-binding pocket (it is not a pseudosubstrate), but it does displace the A-loop to prevent the catalytic domain adopting an active state.

The disposition of the core Inkal sequence (RSRQPLVLGD) in the current structure shows docking in to the substrate-binding pocket (primarily via R-2 and R-4 interactions; Fig. 4c) and the inhibitor chain runs parallel to, and hydrogen bonds with, several main-chain residues of the activation loop in a $\beta$-sheet-like manner (Fig. 3a). Comparison of the PAK4-bound iBox structure
(Fig. 3a,b) with that of the PAK4 AID PAK4 (ref. 33) reveals a common geometry underlying the inhibition. The iBox and AID core sequences resemble a bound consensus substrate peptide; however, the iBox and AID contain a proline residue in place of target serine designated $\operatorname{Ser}(0)$. Analysis of the bond angles of these residues reveals that they fall in the same region of the Ramachandran plot (Fig. 3c). It seems the relative rigidity of proline stabilizes the favourable PAK4-binding conformation of the iBox and AID peptides that mimic bound serine, thus explaining why proline was selected in both during evolution. This is different to most other intramolecular kinase pseudosubstrate sequences, for example, those in the large protein kinase $\mathrm{C}$ family $^{39}$ in which the alanine is present in place of $\operatorname{Ser}(0)$ (RRGA(0)IKQ) in protein kinase C $\alpha$. For the well-known PKIs, an alanine occupies the $\operatorname{Ser}(0)$, and again basic residues at the -2 and -3 positions are critical for kinase domain interaction in the substrate-binding pocket (RRNA(0)IHD) in PKI $\alpha$. The AID and Inkal structures similarly feature Arg-mediated salt bridges that bind an acidic pocket and hydrophobic side-chain interactions at the +2 and +3 positions.

Inka1 binds to PAK4 in a substrate-like manner. Inspection of the three structures (Fig. 4a) suggests a mechanism of phosphate transfer, similar to that proposed for the $\mathrm{PKA}^{40}$ and other protein kinases $^{41}$, with PAK4 Lys442 and Asp440 from the catalytic loop, being close to the ATP $\gamma$-phosphate and Inka1 Pro(0), respectively (Supplementary Fig. 3). To test the model that these inhibitory sequences closely mimic substrate binding 
a

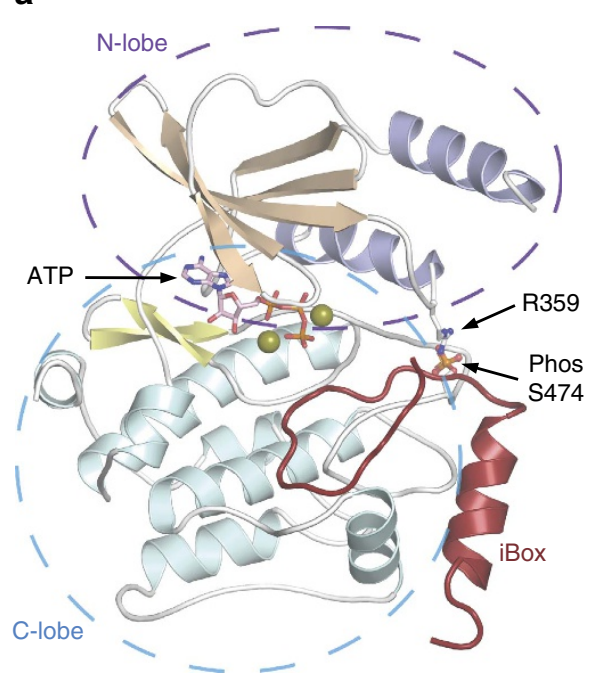

b
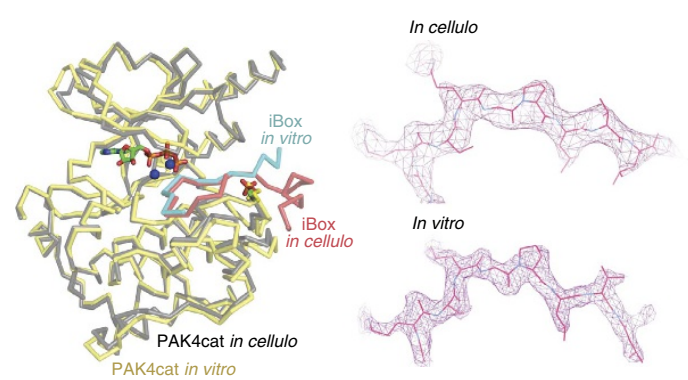

C

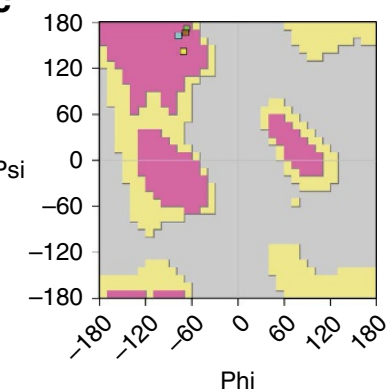

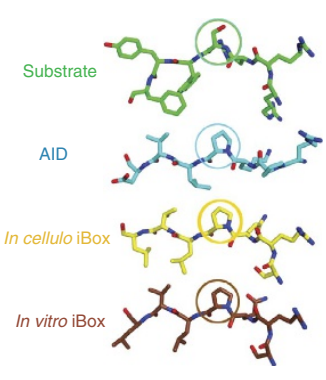

Figure 3 | The in-cellulo X-ray structure of the catalytic domain of PAK4 in complex with Inka1. (a) The X-ray structure of the iBox-PAK4cat complex derived from diffraction of the in-vivo crystals. The typical kinase fold is observed with the iBox (red) binding the PAK4cat close to the phospho-Ser474 (orange), ATP and magnesium ions (mustard). (b) Overlay of in vitro and in vivo PAK4cat:Inka1 complex structure. Comparison between the $\alpha$-carbon traces of Pak4cat:Inka crystallized in vivo (grey and red) and Pak4cat co-crystallized with a synthetic peptide iBox24 (see Fig. 1d). The PAK4cat with iBox24 yielded a structure at $2 \AA$, which was overlaid (backbone of the chains in yellow and cyan). The ATP and two $\mathrm{Mg}^{2+}$, found in the in vivo structure, are represented in stick and sphere format. On the right is the comparison of the electron density maps of the Inka1 core sequence in the two structures. Stereo images of portions of the $2 \mathrm{Fo}-\mathrm{Fc}$ electron density maps contoured at $1.5 \sigma$ and centred at $\mathrm{P}(0)$ in Inka is provided in Supplementary Fig. 6 . (c) Conservation of the bond angles comparing the substrate serine with proline mimetic in Inka1. The local main-chain and side-chain orientation of the substrate serine (SO) and corresponding prolines in the substrate mimetics are as indicated. Values corresponding to these four residues mapped onto the standard Ramachandran plot indicate their similar orientation.

\section{Table 1 | Statistics of data collection and refinementi.}

\begin{tabular}{|c|c|c|}
\hline & In-cellulo PAK4cat:iBox & In-vitro PAK4cat:iBox \\
\hline PDB code & $4 X B R$ & $4 X B U$ \\
\hline Space group & $\mathrm{P}_{3}$ & $\mathrm{P} 4{ }_{1} 2,2$ \\
\hline Resolution $(\AA)$ & $44.2-2.94(3.02-2.94)$ & $29.3-2.06(2.11-2.02)$ \\
\hline$R_{\text {merge }}(\%)$ & $29.4(60.0)$ & $7.4(75.4)$ \\
\hline Completeness (\%) & $97.3(83.4)$ & $100.0(99.9)$ \\
\hline Redundancy & $7.8(2.0)$ & $12.8(12.6)$ \\
\hline \multicolumn{3}{|l|}{ Refinement } \\
\hline Resolution $(\AA)$ (highest-resolution shell) & $20.0-2.94(3.02-2.94)$ & $20.0-2.06(2.11-2.06)$ \\
\hline No. of reflections: working/test & $14702 / 776(906 / 44)$ & 24541/1262 (1599/79) \\
\hline Mean B-factor $\left(\AA^{2}\right)$ PAK4/iBox water ATP/ $\mathrm{Mg}^{2+}$ & $68.9 / 108.9-90.2 / 54.0$ & $38.6 / 50.344 .0-/-$ \\
\hline Ramachandran (\%) favoured/allowed/general/disallowed & $86.1 / 13.6 / 0.4 / 0$ & $92.0 / 8.0 / 0 / 0$ \\
\hline
\end{tabular}

(Supplementary Fig. 4), we replaced Pro(0) with Ser and tested the synthetic 13mer peptides as PAK4 substrates in situ (Fig. 4b). The AID-based peptide was phosphorylated as efficiently as Raf1 Ser338 (ref. 13), but Inka1-derived sequences were significantly better substrates. Alanine scanning substitution showed that the presence of $\operatorname{AID} \operatorname{Arg}(-3)$ or Inkal $\operatorname{Arg}(-2)$ were critical for peptide phosphorylation. These side-chain contacts of Inka1 arginines (Fig. 4c) involve two acidic substrate-binding pocket 
a

PAK4cat + AID (red)

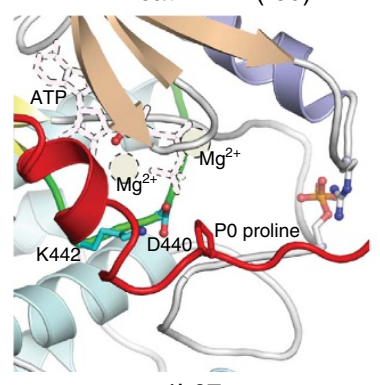

4L67
PAK4cat + iBox (red)

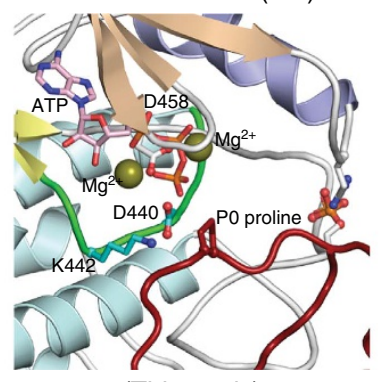

(This study)
PAK4cat + RRRRSWYFDG (pink)

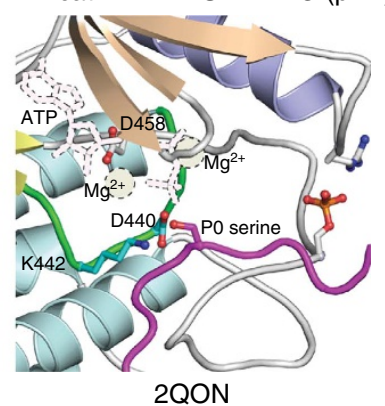

b

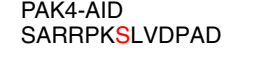

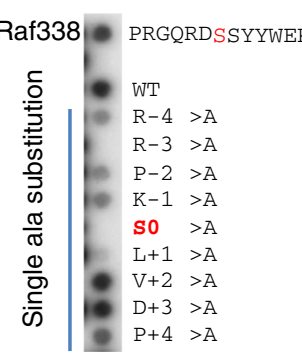

$\left[{ }^{32} \mathrm{P}\right] 3 \mathrm{~h}$ exposure

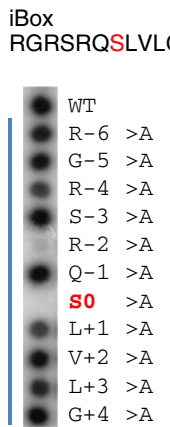

$1.5 \mathrm{~h}$ exposure
iBox-C

SLRSRQSIIYNDV

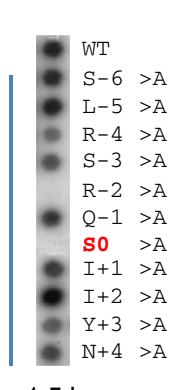

C

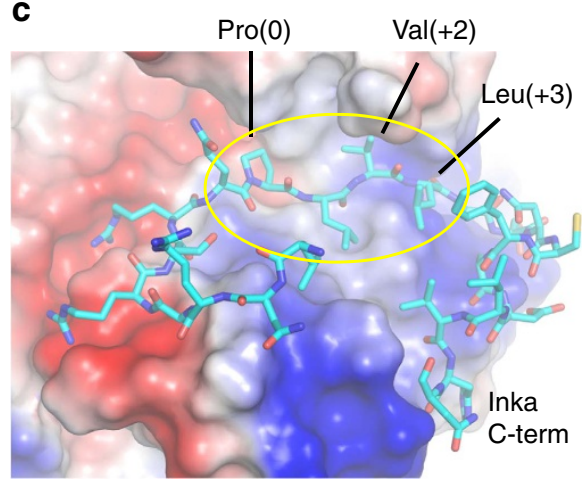

Figure 4 | Inka1 inhibition of PAK4 activity through substrate mimicry. (a) Left-to-right: PAK4:AID (red); the in-cellulo structure of PAK4:iBox (dark red); PAK4:substrate (purple). The inhibitor prolines (PO) are similarly positioned to the serine (SO) of the substrate. (b) To assess the inhibitors as 'supersubstrates' we tested 13aa synthetic peptides with Pro (0)Ser substitutions in an array. The contribution of each side chain to substrate binding was assessed via alanine substitutions. The Ser (0)Ala completely abolished phosphorylation in each case, confirming other serines were not phosphorylated. (c) iBox-PAK4 in-cellulo structure highlighting the cluster of hydrophobic contacts between the Inka1 side chains and the surface of the PAK4 (yellow). The hydrogen bonds are marked in orange.

(circled in Supplementary Fig. 4). Based on the phosphorylation profile both the iBox and iBox-C $\operatorname{Arg}(-4)$ side chains contribute significantly to peptide binding. In the PAK4:Inkal structure the hydroxyl of the Inka1 Ser $(-3)$ side chain forms a hydrogen bond with the Inka1 main chain; however, only in the iBox-C did we note a significant loss of interaction following $\operatorname{Ser}(-3)$ Ala substitution. Changing the $\mathrm{iBox} \operatorname{Leu}(+1)$ and Leu $(+3)$, which lie on a hydrophobic shoulder of the kinase, to alanine affected phosphorylation (Fig. 4b,c) as a result of reducing the side-chain hydrophobicity. Together, these observations explain the conservation of the RSRQPlvl motif among the iBox sequences (Fig. 1, upper case: invariant; lower case: positions non-bulky hydrophobic residues).

The kinase-kinase contacts in Inka1:PAK4 crystals. Inspection of the crystal packing revealed that the crystal is formed by only two types of contacts, both of which are between PAK4cat units (Fig. 5 and Supplementary Movie 1). The crystal packing resembles that obtained for a short (346 residue) isoform of fulllength PAK4 (ref. 14) in which the N-terminal regulatory region is largely disordered, excepting the pseudosubstrate-like peptide (4FIG). In the in-cellulo crystals one set of crystal contacts is formed by the interaction between neighbouring $\mathrm{N}$-lobes that involves the two helices from one N-lobe interacting with the $\beta$-sheet of the adjacent N-lobe, an interaction area of $768 \AA^{2}$. The $\mathrm{N}$-lobe interactions form strands that run the length of the crystal (Fig. 5b and Supplementary Movie 1). The hexagonal packing requires that the $\mathrm{N}$-lobe be in a 'closed' state relative to the C-lobe, which is likely to be achieved through 'clamping' of the Inka1 inhibitory region. Interestingly, the PAK5cat sequence is slightly different at this interface and thus does not generate in cellulo crystals with Inka1. The second set of contacts lies at the threefold axis mediated by the PAK4cat C-lobes involving primarily hydrophobic residues; each C-lobe contributes $576 \AA^{2}$ to this crystal contact (Fig. 5c). Remarkably, the iBox is not involved in crystal contacts and is exposed to the large 80 - $\AA$-diameter central solvent channels that run the length of the crystals (Fig. 5a). These observations thus explain the ability of multiple Inka1 deletion constructs to form crystals with PAK4, as there exists a large space to accommodate the various polypeptides associated with either iBox or iBox-C.

The packing between the N-lobes, as observed in the in-cellulo $\mathrm{P}_{3}$ crystal form, is also reproduced in the in-vitro $\mathrm{P}_{4} 2_{1} 2$ crystal reported here and elsewhere ${ }^{12,14,31,42-44}$, and in an in-vitro $\mathrm{P} 2{ }_{1} 2_{1} 2_{1}$ crystal $^{45,46}$, demonstrating that this interaction is conducive for crystallization. These two crystal forms support a range of apo peptide inhibitors and small-molecule inhibitor complexes with PAK4cat. Furthermore, both the in-cellulo $\mathrm{P}_{3}$ threefold and N-lobe packing interactions are observed in the invitro P3 structures of PAK4 full length, PAK4cat and PAK4cat with bound peptide RPKPLVDP ${ }^{14}$. Thus, the two molecules in the asymmetric unit of the $\mathrm{P} 3$ parent crystals possess the central channel and share similar packing to the single molecule in the asymmetric unit of the in-cellulo $\mathrm{P}_{3}$ crystals. Both $\mathrm{P} 3$ and $\mathrm{P}_{3}$ crystals are able to accommodate larger constructs beyond the PAKcat domain that forms the entire crystal packing, namely the $\mathrm{N}$ terminus of PAK4 and Inkal sequences, respectively.

High-resolution imaging of crystal formation. Based on the crystal structure described above and the available space in the 
a

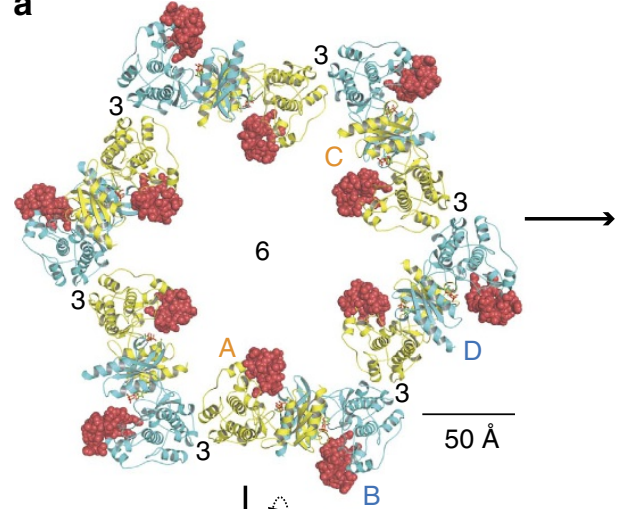

C
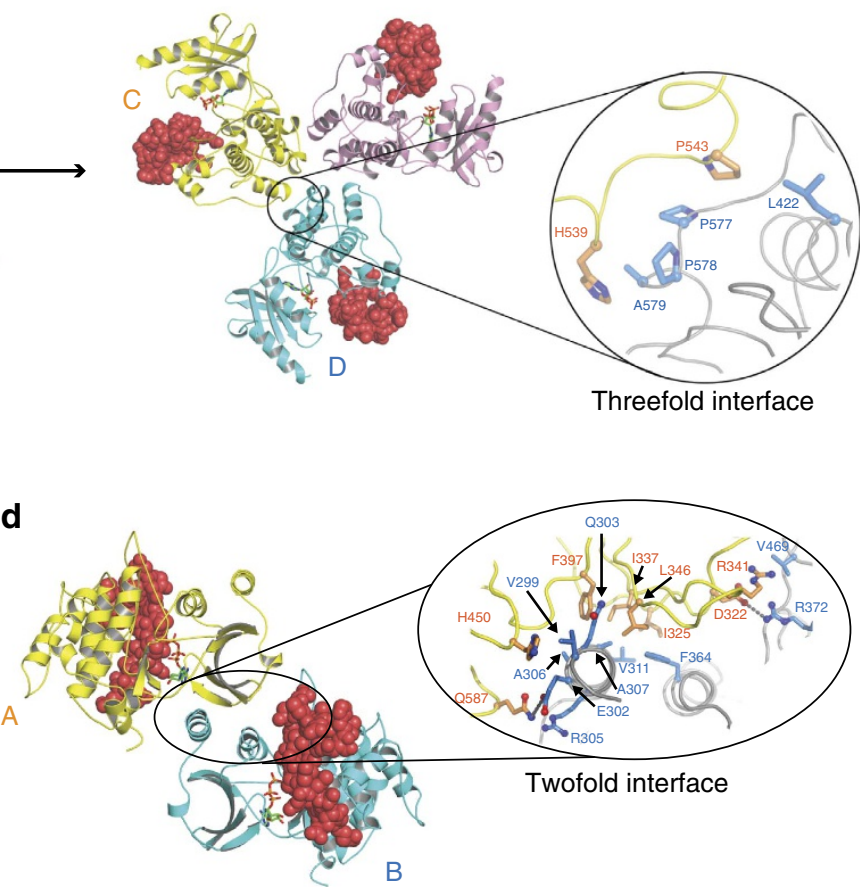

b

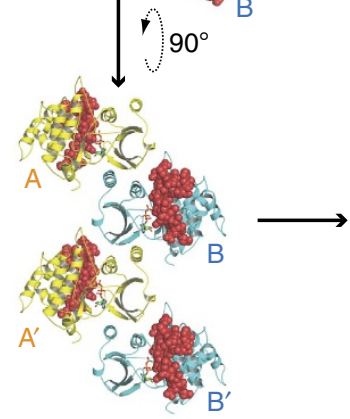

iBox PAK4cat

591

Figure 5 | Crystal packing of the PAK4cat:Inka crystals and the nature of the protein-protein interface. (a) The in-cellulo construct and crystal packing of PAK4cat, which form the channel in the presence of Inka1 (red). The schematic of the construct is similarly coloured. (b) The N-lobes, which form the strands that run along the length of the channel. (c) The threefold axis involves hydrophobic interactions of the C-lobe, primarily involving proline residues as indicated. (d) The twofold interface involves primarily hydrophobic side-chain interactions between the B-subunit (blue) N-lobe $\alpha$-helices including the F364 in the $\alpha$-helix-C, which interacts with the $\beta$-strand sequences. The $\alpha$-helix-C, a conserved feature of protein kinases, co-ordinates PAK4 kinase activity. PAK4cat (alternately yellow and cyan) and iBox (red). Numbers indicate fold axes. This schematic was generated using PyMOL Molecular Graphics System.

lattice, we postulated that hybrid proteins of up to $30 \mathrm{kDa}$ when fused to the iBox might also co-crystallize with PAK4cat in cellulo. Indeed, several GFP-Inkal constructs readily formed co-crystals with PAK4cat (Fig. 6) when expressed in mammalian cells. The crystals formed with GFP-Inka1 and Flag-PAK4cat, allowed for time-lapse analysis of crystal formation (Supplementary Movies 2 and 3). By expressing the membrane marker RFP-CAAX, the plasma membrane could be observed to surround the crystal, as it exceeds the normal dimensions of the cell (Supplementary Movie 5). The co-crystallization of GFP-Inkal and PAK4cat was modelled (Supplementary Movie 6) to demonstrate that there is sufficient scope in the PAK4cat packing to accommodate GFP. At this stage we are unable to confirm that the GFP itself is ordered sufficiently to obtain high-resolution diffraction data. Super-resolution (SIM) imaging of these GFP crystals revealed their underlying hexagonal symmetry (Fig. 6c).

As the Flag-iBox-PAK4 crystal structure contained bound ATP, which is stabilized by the Inkal inhibitory peptide (Fig. 3a), we were interested on the effect of the ATP-competitive PAK4 inhibitor PF-03758309, which binds with $10 \mathrm{nM}$ affinity in vitro ${ }^{45}$. Unexpectedly, GFP-Inka1:HA-PAK4cat co-crystals reproducibly became depleted of GFP signal during the elongation phase in $5 \mu \mathrm{M}$ PF-03758309 (Fig. 6d). Thus, PF-03758309 appears to allow PAK4cat to incorporate with sub-stoichiometric levels of GFP-Inka1, consistent with PF-03758309 either reducing the affinity of GFP-Inka1 or allowing PAK4cat incorporation without Inka1. The average crystal growth along the length (Fig. $6 \mathrm{~d}$ ) was $4.2 \pm 1.2 \mu \mathrm{m} \mathrm{h}^{-1}$, which equates to adding a new layer of crystal lattice every three seconds comprising $\sim 50,000$ protein units (for a crystal with $2 \mu \mathrm{m}$ cross-section). Crystal growth slowed after PF3758309 addition. Based on this analysis, we observed PAK4cat incorporated at both ends of the crystal (Fig. 6d).

\section{Discussion}

The formation of crystals or filaments in mammalian cells is unusual but not unprecedented. Depletion of ATP in cells leads to the assembly of cofilin-actin rods in various cell types including the neurons, and these rods can be purified (Minamide, 2010 \#6413). The enzyme CTP synthase dynamically assembles into macromolecular filaments in bacteria, yeast, Drosophila and mammalian cells; it has recently been shown this might be a physiological response regulated by the non-receptor Cdc42effector kinase DAck in the Drosophila embryo (Strochlic, 2014 \#6412). In these two cases there is evidence that the assemblies play functional role, which has been conserved. It should be noted that PAK4 only forms crystals when it is truncated and one would anticipate such a propensity (in full-length proteins) would be selected against during evolution.

Many human protein kinases are negatively regulated via interaction of the catalytic domain with an $\mathrm{AID}^{47}$, but a few are also targeted by (small) inhibitory proteins, which provide an additional layer of regulation. We have identified Inkal as a potent vertebrate inhibitor of PAK4 with a Ki of $\sim 30 \mathrm{nM}$ (Fig. 1), which has a much higher affinity than the corresponding AID. Inkal contains two copies of the kinase inhibitory domain and both of these small regions of themselves can support PAK4cat 
a

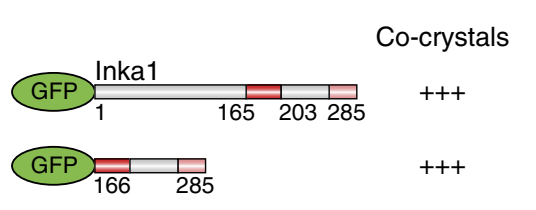

b

Co-crystals

GFP-Inka1
C

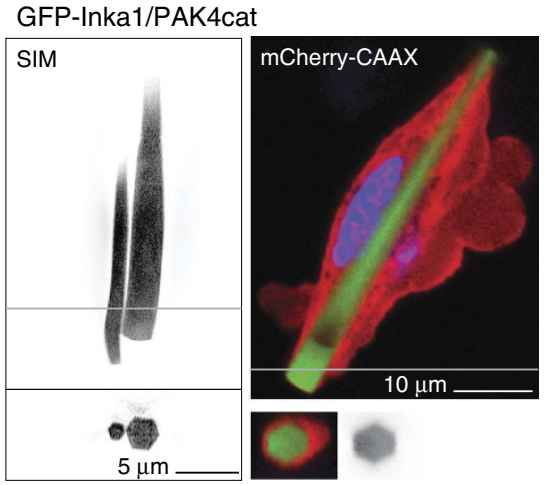

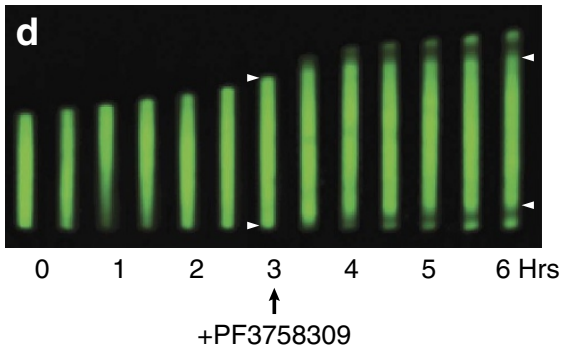
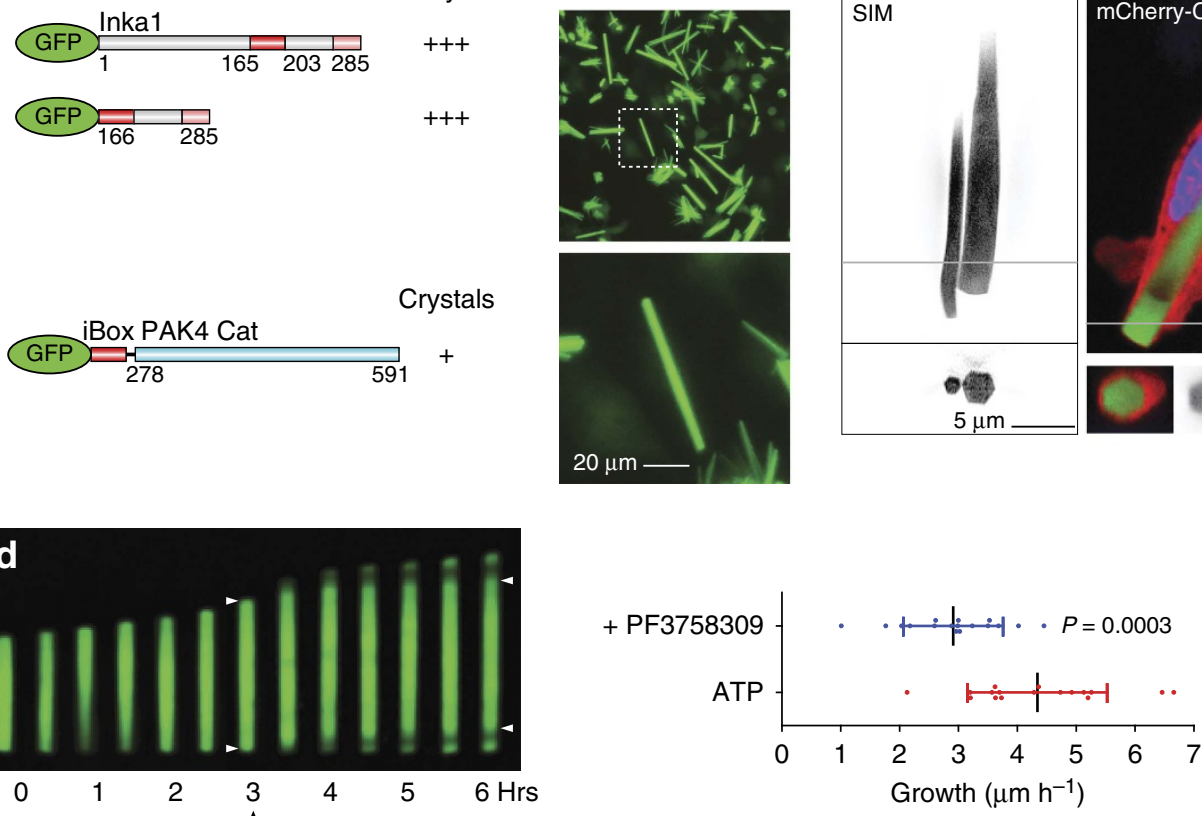

+PF3758309

Figure 6 | Incorporation of GFP into PAK4 crystals and their in-vivo dynamics. (a) Schematic of the fluorescent Inka1 constructs generated and (b) the resultant in cellulo crystals when transfected with PAK4cat. (c) Structured illumination microscopy of a cell containing two crystals (SIM, left) and a single crystal observed by two-channel confocal (right) images of GFP-Inka1:PAK4cat crystals. The cross-sections (line) show the crystal enveloped by membrane (also see Supplementary Movie 4). (d) Effect of addition of PF3758309 (5 $\mu$ M, arrow) on a growing GFP-Inka1:Flag-PAK4cat crystal. GFP incorporation appears to occur at both ends based on the obvious depletion of GFP signal in the growing crystal after PF3758309 is added. The recovery of signal at $1.5 \mathrm{~h}$ after drug addition may be due to drug depletion. Right: the measured growth rates of GFP-Inka1 crystals before and after drug addition ( $n=17$, error bars indicate 1 s.d.).

crystal formation in cells (Fig. 4). To our knowledge, Inka represents one of only six classes of established endogenous protein kinase inhibitors to be uncovered to date. It is likely to be that more remain to be found among the plethora of orphan open reading frames in the human genome; however, none of these different proteins share sequence homology.

Among known endogenous kinase inhibitors, Inka1 represents one of four whose basis of inhibition is understood at the structural level. The three members of the PKIs are proteins of $<100$ residues sharing an N-terminal region of 25 amino acids ${ }^{48}$, which interact with the PKAc catalytic domain as illustrated in Fig. 7. There is evidence that PKI $\gamma$ is required for export of PKA catalytic subunits from the nucleus back to the cytoplasm following activation of PKA in the brain ${ }^{36}$. Based on sequence homology searches, PKI proteins can be found in many invertebrates ( $c f$. K09E9.4 in Caenorhabditis elegans) but not in certain groups such as Drosophila. Two closely related $\mathrm{Ca}^{2+}$ calmodulin-dependent protein kinase II inhibitors (CaM-KIIN) of 78 and 79 amino acids have been characterized and show $\sim 50 \mathrm{nM}$ Ki in vitro ${ }^{49}$.

The best-studied endogenous inhibitors are cyclin-dependent kinase (CDK) inhibitors. The INK4 gene family encodes p16INK4a, p15INK4b, p18INK4c and p19INK4d; all bind to CDK4 and CDK6, and block their association with D-type cyclins ${ }^{50}$. The INK4 inhibitor structure is different from the others described here, in being well folded in the absence of kinase (Fig. 7). The Cip/Kip family members vary widely in size and comprise p21 Cip1/Waf1/Sdi1 (ref. 51), p27Kip1 (ref. 52) and p57Kip2 (ref. 53). These share a conserved N-terminal domain that binds in an extended manner to both cyclins and CDKs, as illustrated in Fig. 7. These proteins, much similar to the JIP family of mitogen-activated protein kinase scaffold proteins, are not stand-alone kinase inhibitors, but rather form a modulatory platform essential for CDK signalling ${ }^{54}$. Finally, the Raf1 and GRK2 inhibitor RKIP is extensively studied and its structure known ${ }^{55}$, but the way by which this protein binds to kinase targets is not known. Mapping studies indicate the non-catalytic domain of Raf1 binds RKIP ${ }^{56}$, which differentiates it from the protein kinase inhibitors shown in Fig. 7.

Both Inka1 and Inka2 are nuclear-localized proteins (Fig. 2), which can be co-immunoprecipitated with Pak4, in particular when the kinase is in an open active state. Inka proteins share sequence homology only in the region that binds to PAK4, which was termed the Inca box ${ }^{17}$; however, we demonstrate that Inka1 (but not Inka2) contains two related functional PAK4 inhibitory modules. There has been some discussion regarding the role of PAK4 in the nucleus, as the kinase undergoes nucleo-cytoplasmic shuttling ${ }^{35}$. The Inka1-LacZ allele expression in mice indicates expression in the cephalic mesenchyme, heart and paraxial mesoderm before E8.5. Subsequently, expression is observed in the migratory neural crest cells; however, the majority of Inka1 - / - mice are viable and fertile $\mathrm{e}^{28}$, pointing to compensation by Inka2. Thus, at this point we infer that Inkal plays a redundant role in regulating PAK4 activity and may well be compensated by Inka2 in mice.

A coral fluorescent protein that forms diffraction-quality micron-sized crystals within mammalian cells has been reported in recent times ${ }^{26}$. These crystals assemble much more quickly and are probably recognized as foreign particles, as they are processed 

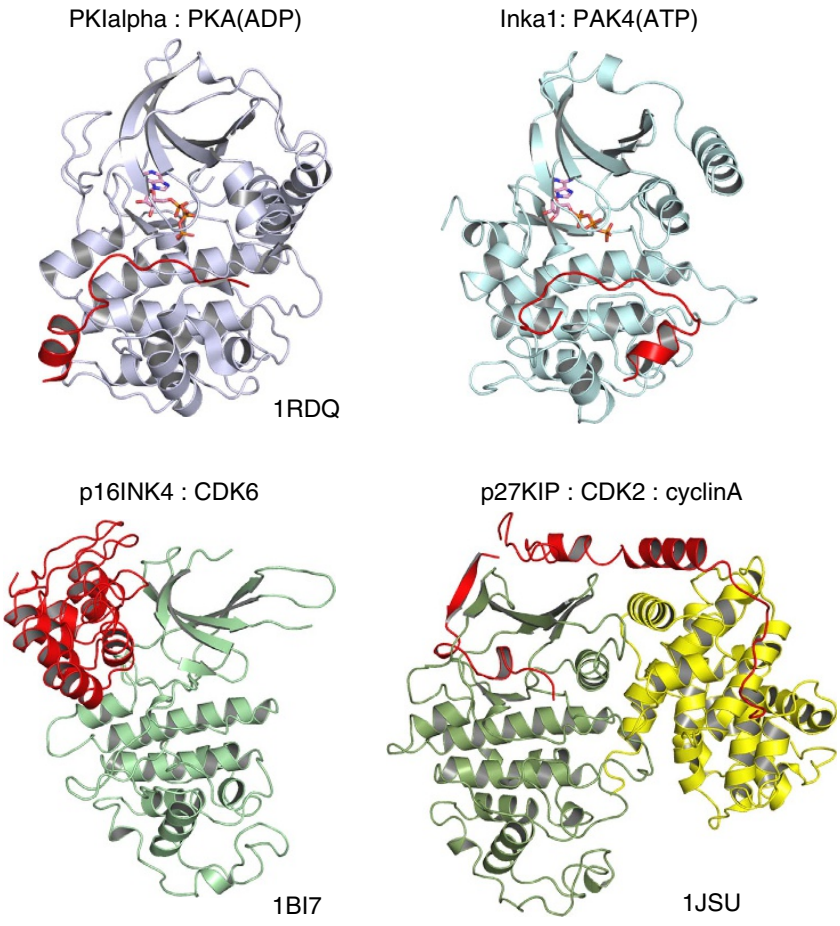

Figure 7 | Representative structures of complexes between known classes of endogenous inhibitors and their target protein kinases. The orientation of the kinase domain (blue or green) in each case is positioned using the conserved secondary helices of the C-lobe. The organization of the inhibitor in each case is shown in red. In the case of p27 KIP, the cyclin A subunit (shown in yellow) provides an important helix to stabilize the CDK2 in an active state. It is noteworthy that the PKI and Inka1 extended region take up similar positions between the $\mathrm{N}$ - and $\mathrm{C}$-lobes, although the helical region of each contacts are very different regions of the $\mathrm{C}$-lobe.

as autophagic cargos. By contrast, our crystals form at a modest pace in the cellular context and grow for 6-16 h (Supplementary Movies 2 and 3), suggesting they are well tolerated in the cytosol over this time period. The complex between PAK4 and Inkal is the first human protein structure to be solved within mammalian cells and, further, multiple constructs of Inkal or fusions to other proteins can be incorporated into the PAK4 crystal lattice (Figs 2 and 6). Crystals have been grown in a variety of mammalian cell types, monkey COS-7 and human HeLa and HEK293 cell lines (Supplementary Fig. 5).

We note parallels to the small-molecule 'crystalline molecular flasks', which have allowed the X-ray structures of the guest molecules to be solved in host frameworks ${ }^{27}$. Stabilizing such guest proteins in a single state probably requires additional engineering of the channel surface, which is currently ongoing. The propensity for mammalian cells to produce single crystals using this system will allow for future structural analysis using microbeam and free-electron laser-based serial femtosecond crystallography ${ }^{57,58}$. Furthermore, the ease with which the crystals can be generated following DNA transformation into mammalian cells suggests uses in other experimental areas, such as for generating high-density in-vivo sensors.

\section{Methods}

Cloning and constructs. All plasmid constructs were generated by PCR-based DNA amplification and inserts completely sequenced. The mammalian pXJ40based vector with Flag, HA and GFP fusion tags contain a standard cytomegalovirus-derived promoter and $\beta$-globin $5^{\prime}$-intron sequence ${ }^{59}$. Inkal constructs were cloned in pXJ-HA (as indicated in Figs 1 and 2) or pXJ-GFP (Fig. 6), whereas
PAK1 and PAK4 were cloned in pXJ-Flag. Flag-GFP-iBox-PAK4cat comprises residues 166-203 of human FAM212A (Inka1), a two-residue linker (Glu$\mathrm{Phe}=\mathrm{EcoRI}$ site) and the kinase catalytic domain of human PAK4 (278-591). For bacterial expression, pGEX4T1 (GE), pET28a (Novagen) and pSY5 (His tagged) were used as expression vectors for Inkal (166-203), PAK1 (1-545) and PAK4 (286-591), respectively. The 13-residue peptide PAK substrate Raf1(S338) PRGQRDSSYYWEI (Raf13p) was used as previously described ${ }^{13}$.

Expression and purification of recombinant proteins. Recombinant proteins were expressed in Escherichia coli BL21-CodonPlus(DE3) (Stratagene) grown at $30^{\circ} \mathrm{C}$. The bacteria were grown to an optical density of $0.6(\mathrm{OD} 600 \mathrm{~nm})$ before induction with $1.0 \mathrm{mM}$ isopropyl- $\beta$-D-thiogalactoside. Induction was carried out for $3 \mathrm{~h}$ at room temperature or $16 \mathrm{~h}$ at $4{ }^{\circ} \mathrm{C}$. Bacterial lysates were purified with $\mathrm{GSH}-$ Sepharose (GE) or nickel Ni-NTA-Agarose (Qiagen) columns, to extract the overexpressed proteins. The recombinant proteins were eluted in $50 \mathrm{mM}$ Tris- $\mathrm{HCl}, \mathrm{pH}$ 8.0, $150 \mathrm{mM} \mathrm{NaCl}, 0.5 \%$ Triton X-100, $10 \%$ glycerol with $5 \mathrm{mM}$ glutathione (for glutathione $S$-transferase (GST) fusions) or $250 \mathrm{mM}$ imidazole (for poly-histidinetagged proteins). With PAK kinases, the elution buffer was supplemented with $1 \mathrm{mM}$ $\mathrm{MgCl}_{2}$. Proteins were diluted and snap frozen in aliquots before use. SDS-PAGE and Coomassie Brilliant Blue staining assessed protein purity to be $>90 \%$.

Transfection of cell culture and immunoprecipitation. Monkey COS-7 cells, human HEK293 and U2OS were grown in DMEM medium with $4,500 \mathrm{mgl}^{-}$ glucose supplemented with $10 \%$ bovine calf serum (Hyclone). HeLa cells were grown in Eagle's MEM medium supplemented with L-glutamine, sodium bicarbonate, sodium pyruvate and $10 \%$ bovine calf serum. Transient transfections were performed with Lipofectamine 2000 according to recommended protocols. Typically, a total of $5 \mu \mathrm{g}$ plasmid DNA was used per $60-\mathrm{mm}$ dish; lysates were harvested $18 \mathrm{~h}$ later in ice-cold lysis buffer $(0.5 \mathrm{ml} ; 25 \mathrm{mM}$ HEPES, pH 7.3, $100 \mathrm{mM} \mathrm{KCl}$, $5 \mathrm{mM} \mathrm{MgCl} 2,20 \mathrm{mM} \beta$-glycerophosphate, $5 \%$ glycerol, $0.5 \%$ Triton X-100, $5 \mathrm{mM}$ dithiothreitol, $0.5 \mathrm{mM}$ phenylmethyl sulfonyl fluoride, $1 \mathrm{mM} \mathrm{Na}_{3} \mathrm{VO}_{4}$ and $1 \times$ protease inhibitor cocktail (Roche)). To test co-immunoprecipitation of proteins, the lysates were clarified by centrifugation $(14,000 g)$ and the clarified lysates were incubated while rolling ( $2 \mathrm{~h}$ ) with $20 \mu \mathrm{l} \mathrm{M} 2$ anti-Flag Sepharose (Sigma-Aldrich, A2220). Rabbit anti-Flag (Sigma-Aldrich, F7425) or horseradish peroxidase-coupled anti-HA (Santa Cruz Biotechnology, sc-7392 HRP, $1 \mu \mathrm{g} \mathrm{ml}^{-1}$ ) were used for western blot analysis.

In-vitro kinase assays. Purified PAK1 or PAK4 $(50 \mathrm{nM}$ in $25-50 \mu \mathrm{l})$ were incubated with $10 \mu \mathrm{M}$ GST-Raf1S338 peptide in $10 \mu \mathrm{M}$ ATP $(2 \mu \mathrm{Ci}$ of $\gamma 32 \mathrm{P}$ ATP $)$ of kinase buffer (25 mM HEPES, pH 7.3, 0.1\% Triton X-100, $50 \mathrm{mM} \mathrm{KCl,} 10 \mathrm{mM}$ $\mathrm{MgCl}_{2}, 1 \mathrm{mM}$ dithiothreitol) at $30^{\circ} \mathrm{C}$ for $20 \mathrm{~min}$. Samples were analysed by SDSPAGE or adsorption of the GST substrate mix onto polyvinylidene difluoride membranes, followed by extensive washing to remove free $\gamma 32 \mathrm{P}$-ATP. The synthetic peptides of $95 \%$ purity, as determined by HPLC and mass spectrometric analyses (GenScript), were soluble in aqueous PBS. Stock solutions $(10 \mathrm{mM})$ were quantified via calculated extinction coefficients and absorbance measurements at $280 \mathrm{~nm}$ and stored at $-80^{\circ} \mathrm{C}$. The diluted peptides were incubated at the indicated concentrations with the kinase on ice $(10 \mathrm{~min})$ before addition of $\gamma 32 \mathrm{P}$ ATP and subsequent incubation at $30^{\circ} \mathrm{C}$. The synthetic peptide array (Jerini Biotools) was phosphorylated in situ as described previously ${ }^{60}$.

Generation and harvesting of intracellular PAK4 crystals. COS-7, HeLa, HEK293 or U2OS cells (35-mm culture dish or glass coverslip) were typically transfected with $2.5 \mu \mathrm{g}$ of each plasmid in $2 \mathrm{ml}$ of media using Lipofectamine 2000 (Invitrogen) or the GenomeONE Neo EX haemagglutinating virus of Japan envelope transfection kit (Cosmo Bio Co. Ltd) as per the manufacturer's protocol. Crystals were observed by phase-contrast microscopy using a $\times 10$ objective (Nikon Eclipse TE300) 1-4 days post transfection. The structure of Flag-iBox-PAK4cat (Figs 2 and 3) was determined from crystals grown in COS-7 cells. The cells were harvested 3 days after transfection by incubating in PBS with $0.125 \%(\mathrm{w} / \mathrm{v})$ trypsin and $25 \%$ (v/v) glycerol (Merck) for $30 \mathrm{~min}$. Individual cells containing single crystals were then mounted in $0.1-0.2 \mathrm{~mm}$ cryoloops (Hampton Research) and flash cooled in liquid nitrogen.

In-cellulo X-ray data collection and structure determination. A $2.95-\AA ̊$ data set was collected at the microfocus beamline I24 of the Diamond Light Source equipped with microapertures, limiting the beam cross-sectional area to $6 \times 6 \mu \mathrm{m}$, at wavelength of $0.9686 \AA$ with a PILATUS3 $6 \mathrm{M}$ detector (DECTRIS, Baden, Switzerland) by merging the diffraction data from five isomorphous crystals. The data were processed with xia2 (ref. 61) and the structure solved by molecular replacement with Phaser ${ }^{62}$, using the coordinates of the catalytic domain of human PAK4 (PDB 4FIE) as the search model. The solution was then built in COOT $^{63}$, refined to completion using REFMAC5 (ref. 64) and validated via the MolProbity web server ${ }^{65}$. Structure figures were generated using PyMOL (The PyMOL Molecular Graphics System, Version 1.3 Schrödinger, LLC). The atomic coordinates and structure factors have been deposited in the Protein Data Bank (PDB 4XBU). 
In-vitro crystallization and $\mathbf{X}$-ray data collection. 6His-PAK4cat protein was purified under standard conditions using a semi-automated Akta system ${ }^{42}$. The crystallization of $6 \mathrm{His}-\mathrm{PAK} 4$ cat was carried by hanging drop method at $5 \mathrm{mg} \mathrm{ml}^{-1}$ with 15 -fold molar excess of the iBox 23 mer synthetic peptide, AEDWTAALLNRGRSRQPLVLGDW, and two times molar excess of ATP. Bipyramidal-shaped crystals grew in $0.1 \mathrm{M}$ Tris- $\mathrm{HCl}, \mathrm{pH} 8.5,12 \%$ PEG 8000 at $25^{\circ} \mathrm{C}$. Crystals were supplemented by $15 \%$ glycerol and flash cooled in liquid nitrogen. X-ray data were collected at a wavelength of $0.9686 \AA$ on I 24 of the Diamond Light Source, and structure solution and refinement carried out as documented for the in-cellulo crystals.

Live cell imaging of crystal growth and SIM and confocal microscopy. The cells were plated at $50 \%$ confluence glass cover slips overnight: plasmid transfection used GFP-iBox-Pak4cat and FLAG-iBox-Pak4cat constructs at a ratio of 4:1, to promote crystal nucleation. The cover slips were transferred to a Chamlide magnetic chamber (Live Cell Instruments, Seoul, Korea) with $5 \% \mathrm{CO}_{2}$ at $37^{\circ} \mathrm{C}$ for live imaging on an Zeiss Axiovert $200 \mathrm{M}$ live-cell imaging with a $\times 10$ objective. We imaged multiple chosen regions for $8 \mathrm{~h}$ at 6 -min intervals. To measure crystal growth rate, we used instead a Nikon Eclipse Ti microscope equipped with spinning disk confocal attachment (Yokogawa CSU-22 module) to avoid photo-damage. The cells were imaged at $\times 601.4$ numerical aperture (NA) objective at 2-min intervals. For SIM and confocal imaging, cells were fixed in non-hardening mounting media (Vectashield). The slides were imaged by Delta vision OMX SIM with a × 1001.4 NA objective. Confocal imaging used an Olympus FV1000 upright system with a $\times 601.42 \mathrm{NA}$ objective. The three-dimensional stacks were analysed by IMARIS software.

\section{References}

1. Abo, A. et al. PAK4, a novel effector for Cdc42Hs, is implicated in the reorganization of the actin cytoskeleton and in the formation of filopodia. EMBO J. 17, 6527-6540 (1998).

2. Jaffer, Z. M. \& Chernoff, J. p21-activated kinases: three more join the Pak. Int. J. Biochem. Cell Biol. 34, 713-717 (2002).

3. Pandey, A. et al. Cloning and characterization of PAK5, a novel member of mammalian p21-activated kinase-II subfamily that is predominantly expressed in brain. Oncogene 21, 3939-3948 (2002).

4. Dart, A. E. \& Wells, C. M. P21-activated kinase 4--not just one of the PAK. Eur. J. Cell Biol. 92, 129-138 (2013).

5. Wells, C. M. \& Jones, G. E. The emerging importance of group II PAKs. Biochem. J. 425, 465-473 (2010)

6. Selamat, W., Tay, P. L., Baskaran, Y. \& Manser, E. The Cdc42 effector kinase PAK4 localizes to cell-cell junctions and contributes to establishing cell polarity. PLoS ONE 10, e0129634 (2015).

7. Wallace, S. W., Durgan, J., Jin, D. \& Hall, A. Cdc42 regulates apical junction formation in human bronchial epithelial cells through PAK4 and Par6B. Mol. Biol. Cell 21, 2996-3006 (2010).

8. Qu, J. et al. PAK4 kinase is essential for embryonic viability and for proper neuronal development. Mol. Cell. Biol. 23, 7122-7133 (2003).

9. Koh, W., Mahan, R. D. \& Davis, G. E. Cdc42- and Rac1-mediated endothelial lumen formation requires Pak2, Pak4 and Par3, and PKC-dependent signaling. J. Cell Sci. 121, 989-1001 (2008)

10. Nekrasova, T. \& Minden, A. Role for p21-activated kinase PAK4 in development of the mammalian heart. Transgenic Res. 21, 797-811 (2012).

11. King, H., Nicholas, N. S. \& Wells, C. M. Role of p-21-activated kinases in cancer progression. Int. Rev. Cell Mol. Biol. 309, 347-387 (2014).

12. Chen, C. et al. Identification of a major determinant for serine-threonine kinase phosphoacceptor specificity. Mol. Cell 53, 140-147 (2014).

13. Baskaran, Y., Ng, Y. W., Selamat, W., Ling, F. T. \& Manser, E. Group I and II mammalian PAKs have different modes of activation by Cdc42. EMBO Rep. 13, 653-659 (2012)

14. Ha, B. H. et al. Type II p21-activated kinases (PAKs) are regulated by an autoinhibitory pseudosubstrate. Proc. Natl Acad. Sci. USA 109, 16107-16112 (2012).

15. Wells, C. M., Abo, A. \& Ridley, A. J. PAK4 is activated via PI3K in HGFstimulated epithelial cells. J. Cell Sci. 115, 3947-3956 (2002).

16. Ng, Y. W. et al. Why an A-loop phospho-mimetic fails to activate PAK1: understanding an inaccessible kinase state by molecular dynamics simulations. Structure 18, 879-890 (2010).

17. Luo, T. et al. Inca: a novel p21-activated kinase-associated protein required for cranial neural crest development. Development 134, 1279-1289 (2007).

18. Dodson, G. \& Steiner, D. The role of assembly in insulin's biosynthesis. Curr. Opin. Struct. Biol. 8, 189-194 (1998).

19. Ackerman, S. J. et al. Charcot-Leyden crystal protein (galectin-10) is not a dual function galectin with lysophospholipase activity but binds a lysophospholipase inhibitor in a novel structural fashion. J. Biol. Chem. 277, 14859-14868 (2002).

20. Charcot, J. M. \& Robin, C. Observation de leucocythemie. C. R. Mem. Soc. Biol. 5, 454-550 (1853)
21. Funke, O. Über das milzvenenblut. Z. Rat. Med. 1, 46 (1851).

22. Gibson, J. F., Ingram, D. J. \& Perutz, M. F. Orientation of the four haem groups in haemoglobin. Nature 178, 906-908 (1956).

23. Redecke, L. et al. Natively inhibited Trypanosoma brucei cathepsin B structure determined by using an X-ray laser. Science 339, 227-230 (2013).

24. Koopmann, R. et al. In vivo protein crystallization opens new routes in structural biology. Nat. Methods 9, 259-262 (2012).

25. Axford, D., Ji, X., Stuart, D. I. \& Sutton, G. In cellulo structure determination of a novel cypovirus polyhedrin. Acta Crystallogr. D Biol. Crystallogr. 70, 1435-1441 (2014).

26. Tsutsui, H. et al. A diffraction-quality protein crystal processed as an autophagic cargo. Mol. Cell 58, 186-193 (2015).

27. Inokuma, Y., Kawano, M. \& Fujita, M. Crystalline molecular flasks. Nat. Chem. 3, 349-358 (2011).

28. Reid, B. S., Sargent, T. D. \& Williams, T. Generation and characterization of a novel neural crest marker allele, Inka1-LacZ, reveals a role for Inkal in mouse neural tube closure. Dev. Dyn. 239, 1188-1196 (2010).

29. Luo, T. et al. Regulatory targets for transcription factor AP2 in Xenopus embryos. Dev. Growth Differ. 47, 403-413 (2005).

30. Eswaran, J., Soundararajan, M., Kumar, R. \& Knapp, S. UnPAKing the class differences among p21-activated kinases. Trends Biochem. Sci. 33, 394-403 (2008).

31. Eswaran, J. et al. Crystal structures of the p21-activated kinases PAK4, PAK5, and PAK6 reveal catalytic domain plasticity of active group II PAKs. Structure 15, 201-213 (2007).

32. Knighton, D. R. et al. 2.0A refined crystal structure of the catalytic subunit of cAMP-dependent protein kinase complexed with a peptide inhibitor and detergent. Acta Crystallogr. D Biol. Crystallogr. 49, 357-361 (1993).

33. Wang, W., Lim, L., Baskaran, Y., Manser, E. \& Song, J. NMR binding and crystal structure reveal that intrinsically-unstructured regulatory domain autoinhibits PAK4 by a mechanism different from that of PAK1. Biochem. Biophys. Res. Commun. 438, 169-174 (2013).

34. Haldeman-Englert, C. R. et al. A 3.1-Mb microdeletion of 3p21.31 associated with cortical blindness, cleft lip, CNS abnormalities, and developmental delay. Eur. J. Med. Genet. 52, 265-268 (2009).

35. Li, Y. et al. Nucleo-cytoplasmic shuttling of PAK4 modulates beta-catenin intracellular translocation and signaling. Biochim. Biophys. Acta 1823, 465-475 2012.

36. Chen, X., Dai, J. C., Orellana, S. A. \& Greenfield, E. M. Endogenous protein kinase inhibitor gamma terminates immediate-early gene expression induced by cAMP-dependent protein kinase (PKA) signaling: termination depends on PKA inactivation rather than PKA export from the nucleus. J. Biol. Chem. 280, 2700-2707 (2005).

37. Zheng, J. et al. Crystal structure of the catalytic subunit of cAMP-dependent protein kinase complexed with MgATP and peptide inhibitor. Biochemistry 32, 2154-2161 (1993).

38. Lei, M. et al. Structure of PAK1 in an autoinhibited conformation reveals a multistage activation switch. Cell 102, 387-397 (2000).

39. Wang, C., Shang, Y., Yu, J. \& Zhang, M. Substrate recognition mechanism of atypical protein kinase Cs revealed by the structure of PKCiota in complex with a substrate peptide from Par-3. Structure 20, 791-801 (2012).

40. Madhusudan, Akamine, P., Xuong, N. H. \& Taylor, S. S. Crystal structure of a transition state mimic of the catalytic subunit of cAMP-dependent protein kinase. Nat. Struct. Biol. 9, 273-277 (2002)

41. Schwartz, P. A. \& Murray, B. W. Protein kinase biochemistry and drug discovery. Bioorg. Chem. 39, 192-210 (2011).

42. Wang, W., Lim, L., Baskaran, Y., Manser, E. \& Song, J. NMR binding and crystal structure reveal that intrinsically-unstructured regulatory domain autoinhibits PAK4 by a mechanism different from that of PAK1. Biochem. Biophys. Res. Commun. 438, 169-174 (2013).

43. Ryu, B. J. et al. Discovery and the structural basis of a novel p21-activated kinase 4 inhibitor. Cancer Lett. 349, 45-50 (2014).

44. Staben, S. T. et al. . Back pocket flexibility provides group II p21-activated kinase (PAK) selectivity for type I $1 / 2$ kinase inhibitors. J. Med. Chem. 57, 1033-1045 (2014).

45. Murray, B. W. et al. Small-molecule p21-activated kinase inhibitor PF-3758309 is a potent inhibitor of oncogenic signaling and tumor growth. Proc. Natl Acad. Sci. USA 107, 9446-9451 (2010).

46. Guo, C. et al. Discovery of pyrroloaminopyrazoles as novel PAK inhibitors J. Med. Chem. 55, 4728-4739 (2012).

47. Huse, M. \& Kuriyan, J. The conformational plasticity of protein kinases. Cell 109, 275-282 (2002)

48. Zheng, L. et al. Cloning and mapping of human PKIB and PKIG, and comparison of tissue expression patterns of three members of the protein kinase inhibitor family, including PKIA. Biochem. J. 349, 403-407 (2000). 
49. Yu, J. S., Chen, W. J., Ni, M. H., Chan, W. H. \& Yang, S. D. Identification of the regulatory autophosphorylation site of autophosphorylation-dependent protein kinase (auto-kinase). Evidence that auto-kinase belongs to a member of the p21-activated kinase family. Biochem. J. 334(Pt 1): 121-131 (1998).

50. Serrano, M., Hannon, G. J. \& Beach, D. A new regulatory motif in cell-cycle control causing specific inhibition of cyclin D/CDK4. Nature 366, 704-707 (1993).

51. Xiong, Y. et al. p21 is a universal inhibitor of cyclin kinases. Nature 366, 701-704 (1993)

52. Toyoshima, H. \& Hunter, T. p27, a novel inhibitor of G1 cyclin-Cdk protein kinase activity, is related to p21. Cell 78, 67-74 (1994).

53. Matsuoka, S. et al. p57KIP2, a structurally distinct member of the p21CIP1 Cdk inhibitor family, is a candidate tumor suppressor gene. Genes Dev. 9, 650-662 (1995).

54. Whitmarsh, A. J. The JIP family of MAPK scaffold proteins. Biochem. Soc. Trans. 34, 828-832 (2006)

55. Rudnitskaya, A. N., Eddy, N. A., Fenteany, G. \& Gascon, J. A. Recognition and reactivity in the binding between Raf kinase inhibitor protein and its smallmolecule inhibitor locostatin. J. Phys. Chem. B 116, 10176-10181 (2012).

56. Corbit, K. C. et al. Activation of Raf-1 signaling by protein kinase C through a mechanism involving Raf kinase inhibitory protein. J. Biol. Chem. 278, 13061-13068 (2003)

57. Schlichting, I. \& Miao, J. Emerging opportunities in structural biology with X-ray free-electron lasers. Curr. Opin. Struct. Biol. 22, 613-626 (2012).

58. Sawaya, M. R. et al. Protein crystal structure obtained at 2.9A resolution from injecting bacterial cells into an X-ray free-electron laser beam. Proc. Natl Acad. Sci. US A 111, 12769-12774 (2014).

59. Manser, E. et al. Expression of constitutively active alpha-PAK reveals effects of the kinase on actin and focal complexes. Mol. Cell. Biol. 17, 1129-1143 (1997).

60. Rennefahrt, U. E. et al. Specificity profiling of Pak kinases allows identification of novel phosphorylation sites. J. Biol. Chem. 282, 15667-15678 (2007).

61. Winter, G., Lobley, C. M. \& Prince, S. M. Decision making in xia2. Acta Crystallogr. D Biol. Crystallogr. 69, 1260-1273 (2013).

62. McCoy, A. J. et al. Phaser crystallographic software. J. Appl. Crystallogr. 40, 658-674 (2007)

63. Emsley, P., Lohkamp, B., Scott, W. G. \& Cowtan, K. Features and development of Coot. Acta Crystallogr. D Biol. Crystallogr. 66, 486-501 (2010).

64. Murshudov, G. N. et al. REFMAC5 for the refinement of macromolecular crystal structures. Acta Crystallogr. D Biol. Crystallogr. 67, 355-367 (2011).

65. Chen, V. B. et al. MolProbity: all-atom structure validation for macromolecular crystallography. Acta Crystallogr. D Biol. Crystallogr. 66, 12-21 (2010).

\section{Acknowledgements}

We thank the Agency for Science, Technology and Research (A*STAR), Singapore, for support. We acknowledge the Joint Centre for Structural Biology, Singapore, which is supported by grants from Nanyang Technological University and the Biomedical Research Council (BMRC) of $A^{\star S T A R}$, for providing research facilities. We thank Diamond Light Source (proposal MX8423) for beamtime, Danny Axford for help with the microapertures and Graeme Winter for with help with data processing. The Wellcome Trust provided administrative support $(090532 / \mathrm{Z} / 09 / \mathrm{Z})$ to J.M.G. Part of this work was funded by the GSK-IMCB Research fund

\section{Author contributions}

E.M., Y.B. and B.C.R. conceived the study and wrote the manuscript with input from all authors. E.M., Y.B. and B.C.R. designed experiments. Y.B. designed and generated cDNA plasmid constructs, and carried out all mammalian cell culture experiments. K.C.A. and W.L.C. performed protein purification and in-vitro crystallization experiments. K.C.Y., R.C.R. and J.M.G. carried out X-ray data collection and structure refinement. P.V.A. collected and processed live-cell and super-resolution imaging of PAK4 crystals.

\section{Additional information}

Accession codes: Atomic coordinates and structure factors files have been deposited in the Protein Data Bank under accession code 4 XBU.

Supplementary Information accompanies this paper at http://www.nature.com/ naturecommunications

Competing financial interests: The authors declare no competing financial interests.

Reprints and permission information is available online at http://npg.nature.com/ reprintsandpermissions/

How to cite this article: Baskaran, Y. et al. An in cellulo-derived structure of PAK4 in complex with its inhibitor Inka1. Nat. Commun. 6:8681 doi: 10.1038/ncomms9681 (2015)

(c) (i) This work is licensed under a Creative Commons Attribution 4.0 International License. The images or other third party material in this article are included in the article's Creative Commons license, unless indicated otherwise in the credit line; if the material is not included under the Creative Commons license, users will need to obtain permission from the license holder to reproduce the material. To view a copy of this license, visit http://creativecommons.org/licenses/by/4.0/ 